\title{
La calidad del aire en la Zona Metropolitana de la Ciudad de México y recomendaciones para mejorarla considerando conceptos básicos de ingeniería ambiental
}

\author{
H. Bravo-Alvarez, R. Sosa-Echeverría, P. Sánchez-Alvarez y M. Jaimes-Palomera \\ Centro de Ciencias de la Atmósfera, UNAM \\ Sección de Contaminación Ambiental \\ E-mail:hbravo@servidor.unam.mx
}

(recibido: septiembre de 2001 ; aceptado: mayo de 2002)

\section{Resumen}

La contaminación atmosférica en la Zona Metropolitana de la Ciudad de México (ZMCM), se hizo evidente en la década de los años sesenta y, a la fecha, ha alcanzado dimensiones considerables. En este trabajo se presenta la evolución del problema de contaminación atmosférica en la ZMCM, enfocándolo a contaminantes criterio como partículas y ozono, y contaminantes tóxicos como el benceno. Asimismo, se exponen experiencias exitosas y las que han fracasado en busca de una mejor calidad del aire para la ZMCM; es recomendable que dichas experiencias sean consideradas en otras ciudades de nuestro país, así como en otros países en vías de desarrollo, ofreciéndoles la importancia debida. Deben considerarse los conceptos básicos de la ingeniería ambiental como son la prevención, la minimización y el control de la contaminación en la fuente, ya que mandatos del ejecutivo tendientes a reducir niveles de bióxido de azufre y de plomo en la atmósfera metropolitana trajeron, por falta de la infraestructura necesaria, un drástico incremento en los niveles, frecuencia y duración de las concentraciones de ozono en la atmósfera.

Descriptores: calidad del aire, Ciudad de México, partículas, ozono, benceno.

\footnotetext{
Abstract

The atmospheric pollution in the Mexico City Metropolitan Zone (MCMZ), was detected during the 1960 s and presents now a days considerable dimensions. The atmospheric pollution problem evolution in the MCMZ is presented in this paper, considering criteria pollutants as particles and ozone, and hazardous air pollutants as benzene. Successful and unsuccessful experiences looking for improve the air quality in the MCMZ are evaluated. These experiences should be account for in other Mexican cities, as well as in developing countries. The MCMZ air quality evolution is an important case where implemented strategies in order to reduce sulphur dioxide and lead, resulted an increase in levels, frequency and duration of ozone concentrations in the atmosphere. Basic concepts of environmental engineering: prevention, minimization and control, as well as pollution control in the source, must be considered.
} 


\section{Introducción}

La Zona Metropolitana de la Ciudad de México (ZMCM), está localizada en la parte suroeste de la Cuenca de México a una altitud de 2,240 mts. sobre el nivel del mar. La situación geográfica de la Cuenca no permite, la mayor parte del tiempo, la libre circulación del viento y una buena ventilación, ya que presenta un patrón diurno dominante en la dirección noreste y noroeste. La zona industrial, localizada en el sector norte de la ZMCM engloba más del $30 \%$ del total de la industria nacional y cuenta con aproximadamente 20 millones de habitantes que representan más de la quinta parte de la población del país, aparte de contar con cerca de 30000 industrias, 12000 instalaciones de servicio y más de 3 millones de vehículos en circulación. En la ZMCM el problema de contaminación atmosférica ha alcanzado niveles críticos en muy poco tiempo, resultando un grave riesgo para la salud de sus habitantes.

Es necesario reconocer que no existen soluciones mágicas ni medidas correctivas únicas, por lo que todas las acciones de control pueden y deben ser aditivas, ninguna sustituye a otra y aunque en apariencia su eficiencia sea baja, la suma de todas ellas podrá mejorar definitivamente la calidad del aire. Por lo anterior, se deberán adoptar políticas agresivas de corto y largo plazo que permitan verificar separadamente y en conjunto la eficiencia de cada una de ellas, así como darle continuidad a los programas que hayan probado su beneficio.

Teniendo documentado que la ZMCM sufre problemas críticos de contaminación atmosférica por ozono y partículas suspendidas, también se han realizado esfuerzos en la evaluación y control de compuestos orgánicos volátiles (COVs), por ser precursores de ozono; sin embargo, al presentarse en la ZMCM emisiones potenciales importantes de contaminantes atmosféricos tóxicos, se deben considerar a varios COVs por su toxicidad, como es el caso del benceno, para el cual existe suficiente evidencia sobre sus efectos cancerígenos en humanos.

\section{Antecedentes}

El problema de la contaminación atmosférica en la ZMCM se hizo evidente en la década de los años sesenta y a la fecha ha alcanzado dimensiones considerables.

No obstante, cabe mencionar que desde esos años setenta se han aplicado algunas acciones para atender este problema. En 1978, el Departamento del Distrito Federal en coordinación con la extinta Subsecretaría de Mejoramiento del Ambiente (SSA), llevó a cabo la Primera Reunión de Expertos, en donde invitaron especialistas de diversos países y académicos mexicanos, con el fin de evaluar los programas y acciones que debían llevarse a cabo para controlar el problema del deterioro atmosférico originado por oxidantes fotoquímicos y otros contaminantes.

En 1979, en una reunión del gabinete presidencial organizada por el Jefe del Ejecutivo Federal, se firma el acuerdo que establece el Programa Coordinado para el Mejoramiento de la Calidad del Aire en el Valle de México (1979-1982).

Años más tarde, en 1986, y como resultado de un mandato presidencial, se redujo rápidamente el contenido de tetraetilo de plomo en las gasolinas, y se eliminó el combustoleo pesado como combustible en las grandes fuentes de combustión dentro de la ZMCM, sustituyéndose por gas natural. Esto originó resultados con una falta de planeación, infraestructura y una estrategia adecuada, ya que dio lugar a los altos valores de ozono que actualmente se presentan en la ZMCM. La recién creada Subsecretaría de Ecología (SEDUE 1987-1988), propone a través de un acuerdo Presidencial, las "Veintiún medidas para controlar la contaminación en el Valle de México", mismas que meses después se amplían en el programa "Cien acciones necesarias en ecología alcance nacional", que cubren además otros problemas ambientales.

Para 1989, nuevamente se logra la participación de expertos mexicanos y extranjeros, por lo que se presenta a la opinión pública el Programa Integral contra la Contaminación Atmosférica en la Zona Metropolitana del Valle de México (PICCA), contando con el apoyo económico sustancial de diversas agencias internacionales (DDF, 1989), pero sin establecer estrategias de control que tiendan a resolver de fondo este problema.

Desde 1987 a la fecha, se rebasó la norma de calidad del aire para ozono en un promedio de 330 
días al año (PROAIRE, 1996; Bravo et al., 1998 a y 1998 b). Además, los datos más recientes muestran una tendencia a rebasar la norma anual de partículas, tanto de Partículas Suspendidas Totales (PST) como fracción respirable (PM10) (INE, 1996).

Para hacer frente a este problema, en marzo de 1996 se presentó a la opinión pública el Programa para el Mejoramiento de la Calidad del Aire en el Valle de México, 1995-2000 (PROAIRE, 1996), preparado por la Comisión para la Prevención y Control de la Contaminación Ambiental en la Zona Metropolitana del Valle de México.

Este programa es uno más de los que a través de las últimas dos décadas ha establecido el Gobierno Federal en conjunto con las autoridades locales, en dicho programa se reconoce un incremento dramático en el comportamiento de las concentraciones máximas diarias de ozono a partir de 1986. Se puede señalar que el PROAIRE no ha cumplido con las expectativas creadas, ya que a la fecha no se ha logrado el efectivo control en el principal problema de contaminación atmosférica del área, que es la elevada concentración de ozono con alta frecuencia y duración, así como las partículas suspendidas totales y partículas fracción respirable (Aldana et al., 1999 a y 1999 b).

\section{Calidad del aire en la ZMCM}

Como se dijo anteriormente, el problema de la contaminación atmosférica en la ZMCM se hizo evidente desde los años sesenta, cuando los contaminantes que excedían las normas de calidad del aire eran partículas, bióxido de azufre y plomo. Actualmente, este problema ha alcanzado dimensiones criticas, ya que los niveles de concentración máxima, excedencias y frecuencia de ozono y partículas suspendidas sobrepasan las Normas Mexicanas de Calidad del Aire. De manera esquemática se presenta la evolución de la contaminación atmosférica en la ZMCM en la figura 1 (Bravo et al., 2001 a).

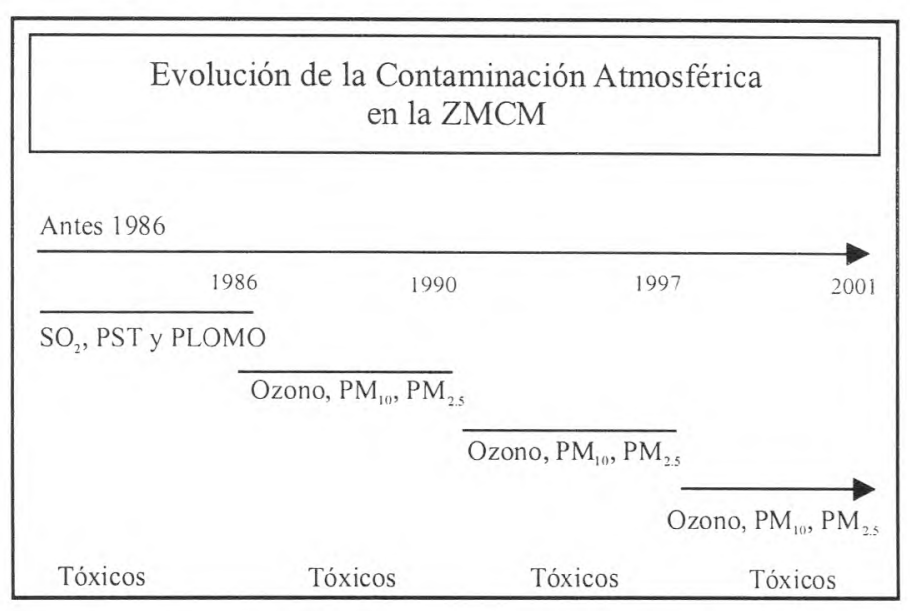

Figura 1. Evolución de la contaminación atmósferica en la ZMCM

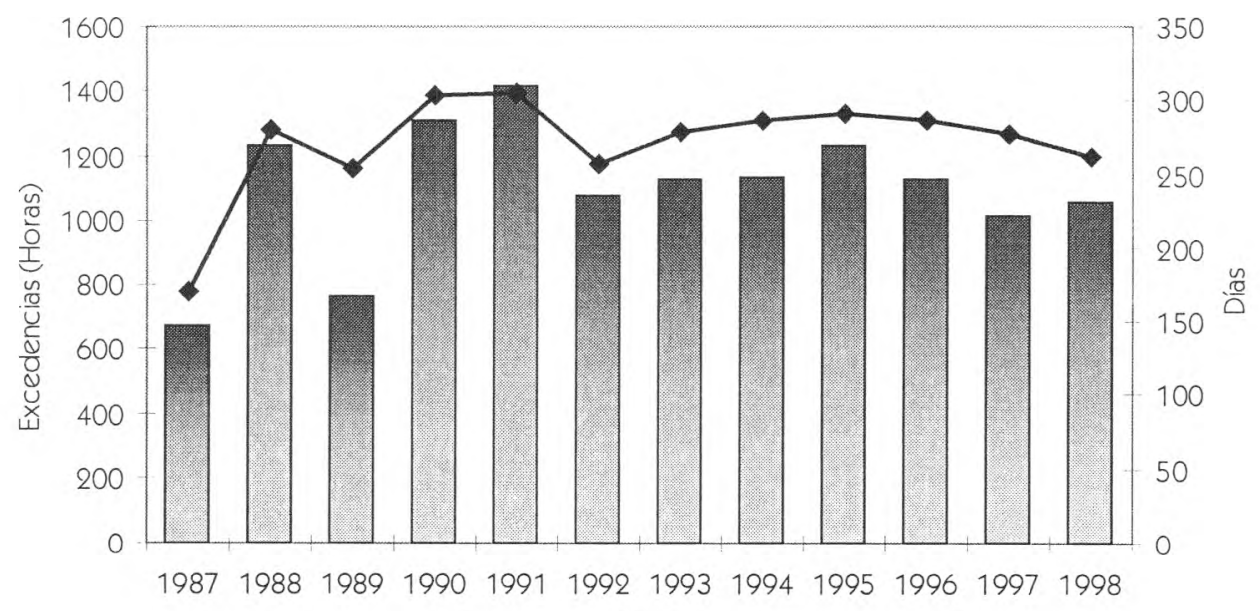

Años

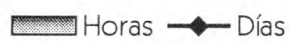

Figura 2. Número de excedencias y número de días arriba de la norma mexicana de calidad del aire para ozono en la estación Pedregal en la Ciudad de México 


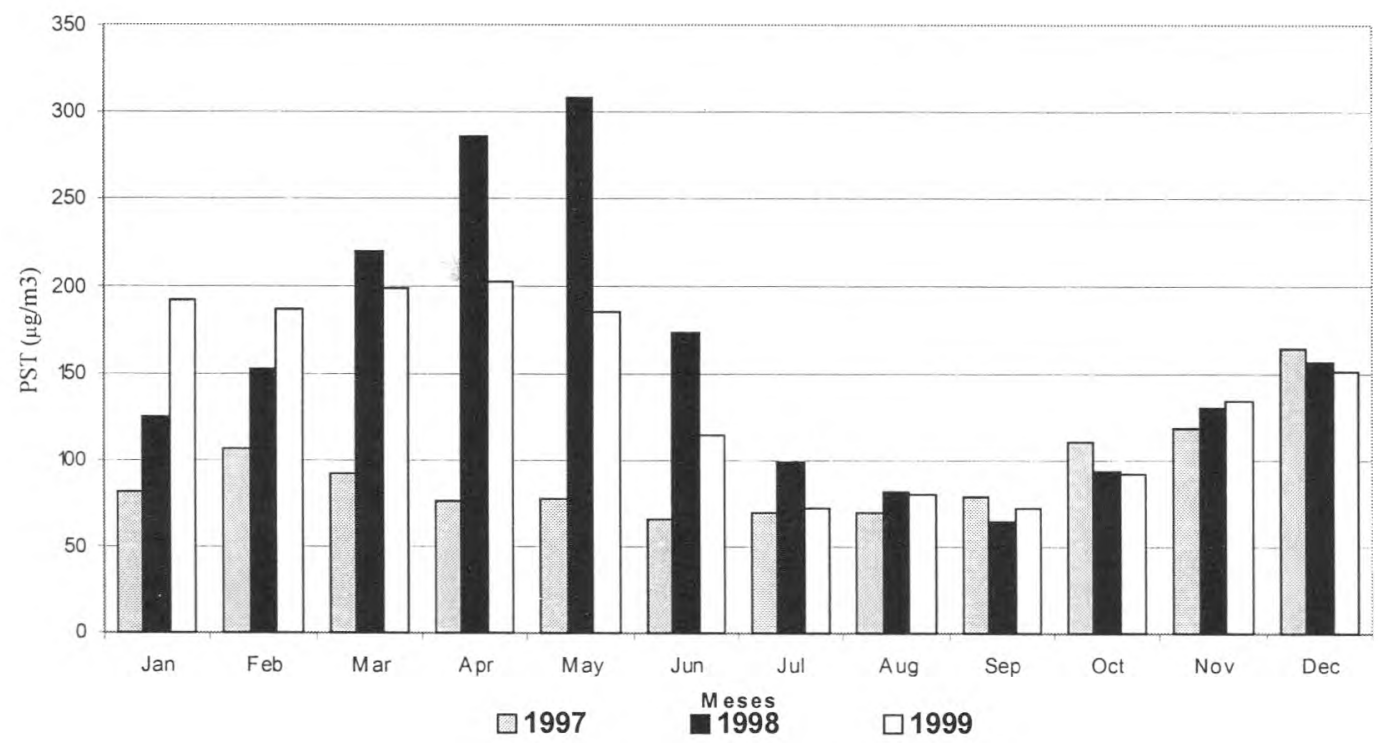

Figura 3. Promedio mensual de la concentración de PST en la estación Pedregal de la Ciudad de México

A partir de 1990 se ha rebasado en la ZMCM la norma de calidad del aire para ozono $(0.11 \mathrm{ppm}$, promedio horario máximo, no más de una vez al año) alrededor de 330 días al año. Tan solo en la estación Pedregal de la Red Automática de Monitoreo Atmosférico (RAMA), en 1991 se presentaron más de 300 días y más de 1400 horas por arriba de la norma de calidad del aire para ozono (Figura 2).

En cuanto a partículas, los datos más recientes exponen una tendencia a rebasar su norma de calidad del aire anual, tanto de partículas suspendidas totales (PST), como de partículas menores a diez micrómetros ( $\mathrm{PM}_{10}$ ) (Vega et al., 1998; INE, 1996). La calidad del aire por partículas es impactada de manera importante por las emisiones de incendios forestales, tal situación fue grave en la ZMCM durante el período de secas de 1998. En la figura 3 se presentan las concentraciones mensuales promedio de PST para la estación Pedregal de la RAMA con el fin de demostrar la influencia de los incendios forestales en la calidad del aire con relación a las partículas; en esta figura se observa un incremento entre el $200 \%$ y $300 \%$ de las concentraciones de PST en 1998, comparado con 1997 durante los meses con un mayor número de incendios forestales (marzo-mayo) (Bravo et al., 1999 y 2001 c).

Otros contaminantes significativos en la atmósfera de la ZMCM son los COVs, por ello la importancia de su estudio consiste no solamente en su papel determinante como precursores de ozono, sino también en el potencial de toxicidad que tiene cada compuesto, dependiendo de sus características, por ejemplo el benceno que es un compuesto poco reactivo; por ello el interés de su control radica más en su toxicidad que como precursor de ozono.

La información más reciente sobre el benceno como causa de cáncer en humanos, está basada en un número determinado de casos de leucemia en personas expuestas. Estos estudios datan de 1928 , y determinaron que el benceno es causante de todo tipo de leucemia, cáncer en los pulmones, hígado, estómago, esófago, faringe e intestino entre otros (Mehlman, 1994).

Debido a los efectos genotóxicos y cancerígenos del benceno, resulta imposible determinar una concentración a la cual la población puede estar expuesta sin riesgo, y más difícil aún, es que existan los métodos para la detección de niveles tan bajos. Sin embargo, algunos países han adoptado un estándar de calidad del aire para fines prácticos que considera concentraciones relativamente pequeñas, pero que a su vez puedan ser detectadas con la metodología de muestreo y análisis existente. En el Reino Unido y Japón se cuenta con un estándar de calidad del aire de 1 ppb como promedio anual (EPAQS, 1991 y EA, 1999). Estudios realizados 
recientemente han reportado niveles 10 veces superiores de este compuesto en gasolinerias en comparación con áreas escolares o unidades habitacionales (Bravo et al., 2001 b y Sosa, 2001). Las concentraciones de benceno encontradas en tres sitios estudiados en la zona suroeste de la ZMCM se presentan en la figura 4.

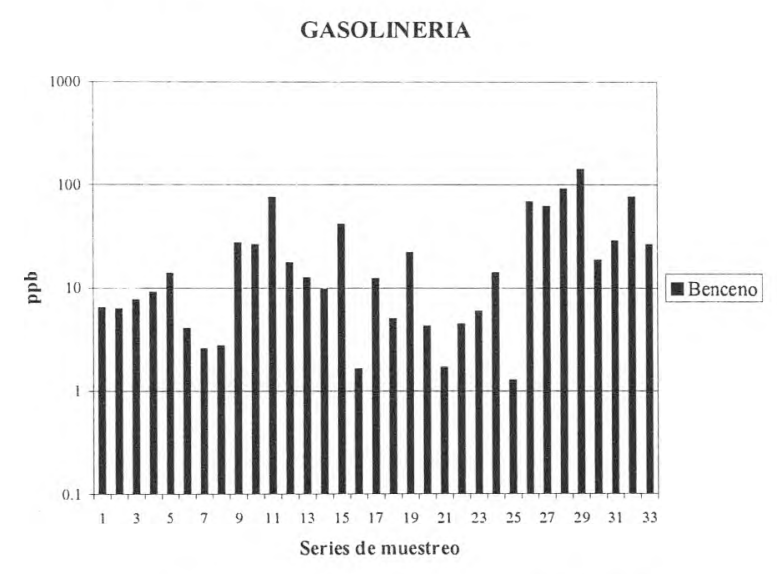

CENTRO DE CIENCIAS DE LA ATMÓSFERA

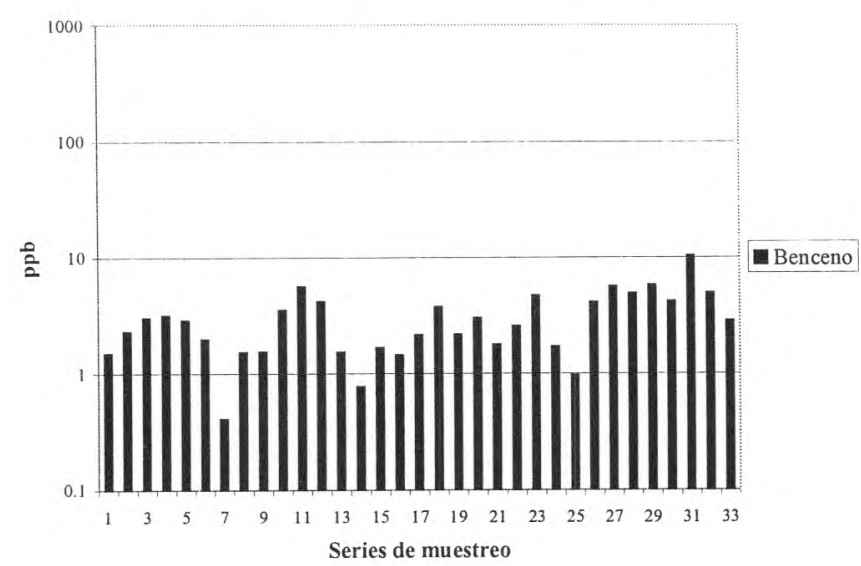

UNIDAD HABITACIONAL

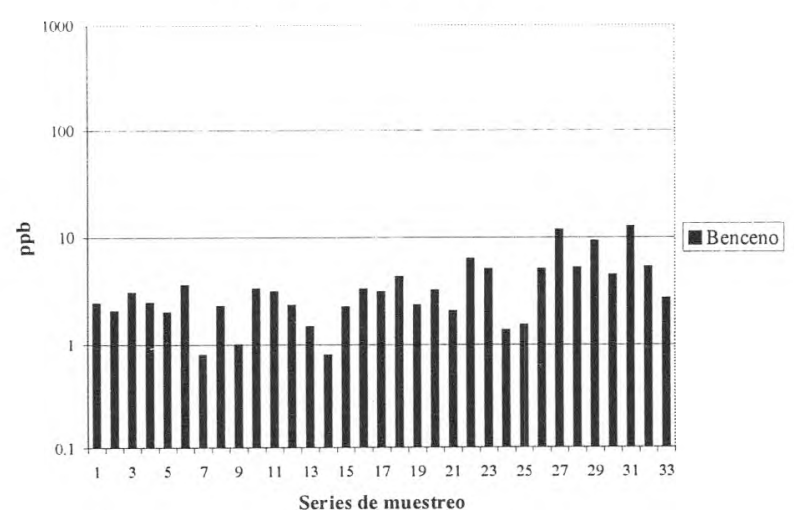

Figura 4. Concentraciones de benceno en tres sitios de muestreo en la ZMCM

\section{Acciones para mejorar la calidad del aire}

El crecimiento del deterioro ambiental, la contaminación atmosférica en la Ciudad de México y la contaminación de lagos y ríos no fue de interés para las autoridades, sino hasta 1971 cuando se promulgó la primera ley para prevenir y controlar la contaminación ambiental. Posteriormente, el ingreso para controlar la contaminación no produce por más de una década beneficios comprobables, por lo que las autoridades sanitarias niegan sistemáticamente los efectos en la salud.

Durante los años ochenta el mayor interés oficial para atacar la contaminación atmosférica se presentó en la reducción de los niveles de plomo, partículas y bióxido de azufre; asimismo, por mandato presidencial se cambiaron en la ZMCM las características reductoras de la atmósfera, a una con carácter oxidante (ozono y partículas). A mediados de los noventa, surge la necesidad de combatir el problema, analizando sus causas, que en el caso del ozono consistió en reconocer la necesidad del estudio de los COVs como precursores, además de los óxidos de nitrógeno.

En 1996 se establece el Programa para Mejorar la Calidad del Aire en el Valle de México, conocido como PROAIRE, el cual se tomará como punto de partida para posteriormente generar comentarios y recomendaciones al respecto. Este programa tiene como metas generales las siguientes:

1. Industria limpia: Reducción de emisiones por unidad de valor agregado en la industria y establecimientos de servicio.

2. Vehículos limpios: Disminución de emisiones por kilómetro recorrido.

3. Nuevo orden urbano y transporte limpio: Regulación del total de kilómetros recorridos por los vehículos automotores.

4. Recuperación ecológica: Abatimiento de la erosión

\section{Resultados}

A pesar que se han realizado esfuerzos y se han destinado numerosos recursos para reducir el problema de la contaminación atmosférica en la ZMCM, es fácil demostrar el poco éxito obtenido a 
la fecha, con respecto a ozono. Esto se debe entre otros factores a situaciones como las siguientes:

a) La falta de verdadera voluntad política para resolver los problemas de fondo, ya que la contaminación o el deterioro ambiental sólo es la consecuencia de dificultades más graves por las que atraviesa nuestra ciudad.

b) Los recursos asignados para llevar a cabo las acciones propuestas han sido escasos, inoportunos y mal aplicados.

c) La carencia de evaluaciones técnicas serias y completas de las acciones propuestas, o la instrumentación precipitada, incluso en contra de la opinión de especialistas que en su momento se opusieron a ellas por considerarlas científicamente no fundamentadas.

d) La falta de seguimiento de cada una de las medidas.

A este respecto se podrían mencionar ejemplos como:

1. La disminución del contenido de plomo en la gasolina en 1986 y la sustitución del combustoleo en las dos plantas termoeléctricas de la ZMCM por gas natural, así como el cierre de las instalaciones de Loreto y Peña Pobre, medidas enfocadas a reducir los niveles de óxidos de azufre, partículas y plomo, pero cuyo efecto colateral fue el incremento de las concentraciones de precursores de ozono. Dichos aspectos fueron advertidos y señalados a las autoridades antes de la instrumentación de las medidas, cuyos resultados se han demostrado en estudios posteriores a la implantación de tales acciones (Bravo et al., 1987, 1988, 1990; Chow, 1990).

2. El uso de gasolinas oxigenadas en vehículos sin convertidor catalítico que redujo sustancialmente la emisión de monóxido de carbono y de plomo, incrementando asimismo los niveles de contaminantes precursores de ozono (Bravo et al., 1997 b, 2000 a y 2000 b).

3. El error de transformar una buena medida con carácter de temporal a permanente (un día sin auto) y el consecuente incremento de vehículos en circulación.
4. El cierre de una importante fuente de reductores atmosféricos como lo fue la Refinería 18 de Marzo, medida que como consecuencia modificó el comportamiento reactivo de la atmósfera de la ZMCM.

Se puede afirmar que en las acciones realizadas hasta ahora, no se ha considerado la complejidad de la química atmosférica de la ZMCM, por lo cual ha resultado un incremento neto de las concentraciones de ozono, representando un serio problema de salud pública (Bravo et al., 1993, 1997 a, 1998 a).

\section{Conclusiones y recomendaciones}

Considerando que se ha logrado una mejoría en la calidad de los combustibles industriales con respecto a su contenido de azufre, al adquirir niveles aceptables en las concentraciones de bióxido de azufre en la atmósfera, los esfuerzos de control deberán enfocarse a la reducción de precursores de ozono.

Asimismo, se deben establecer normas con límites de emisión de hidrocarburos reactivos y óxidos de nitrógeno en establecimientos industriales y de servicio.

Es importante que las autoridades locales tengan la facultad para la aplicación de la normatividad ambiental correspondiente. También es necesario que las autoridades hacendarias establezcan mecanismos crediticios directos para la adquisición de equipo de control de emisiones o modernización de procesos, tomando en cuenta que la situación económica del país impide a la pequeña y mediana industria contar con este tipo de recursos.

Se debe llevar a cabo el inventario de contaminantes tóxicos atmosféricos en la ZMCM, y en especial el de benceno, lo que sería de gran ayuda para identificar las fuentes, y así, entender la presencia de este contaminante en la atmósfera para fundamentar eficientemente las estrategias de control.

Se debe evaluar la factibilidad de establecer en México una norma de calidad del aire para benceno, aceptando que no se podría ofrecer una absoluta 
seguridad de protección a la salud, lo cual daría como resultado que esta norma se haga más estricta. Es decir, se manejaría el concepto de meta de calidad del aire que consiste en reducir paulatinamente los niveles de contaminación atmosférica para mejorar la calidad del aire.

La disminución de emisiones por fuentes móviles contempla medidas de control desde las más sencillas hasta las más complejas, como el uso de dispositivos de control de emisiones en vehículos automotores, el uso de combustibles adecuados para las condiciones de la ZMCM y su parque vehicular, mejorar la vialidad, incentivar el empleo de transporte público de alta capacidad, etc.

La disminución de emisiones por evaporación en estaciones de servicio, se ha realizado de manera continua y progresiva en la ZMCM desde 1995; sin embargo, al presentarse niveles críticos de benceno, ha sido necesario verificar las condiciones de operación de estos sistemas de control de emisiones evaporativas y para requerir mayor eficiencia y disminuir la exposición personal.

La existencia en la ZMCM de un parque vehicular con muchos años de antigüedad, ocasiona que los avances tecnológicos de reducción de emisiones sean inaplicables o poco eficientes. Cabe mencionar que más del $60 \%$ de los vehículos que circulan en la ZMCM carecen de convertidor catalítico.

La optimización del consumo de combustibles de calidad adecuada debe ser una de las estrategias a seguir, por lo que se deben evaluar sus características con base a normas de calidad adecuadas, principalmente en la reactividad y toxicidad de sus emisiones, antes de su distribución, tomando en cuenta las características del parque vehicular y las condiciones de la Ciudad de México.

Es importante llevar a cabo un programa de vigilancia sobre la calidad de los combustibles por la autoridad ambiental del GDF, y un laboratorio independiente del sector gobierno.

Se deben hacer obligatorios los estudios de evaluación de impacto ambiental, analizando las medidas que puedan afectar la calidad del aire de la ZMCM, tales como la introducción de nuevos combustibles, programas restrictivos del tránsito vehicular, cambios de horario como el de verano, etc.

Asimismo, llevar acabo una evaluación transparente y objetiva del PROAIRE, considerando, tanto sus aciertos como sus errores, para que su aplicación resulte con una calidad de aire satisfactoria en la ZMCM

La participación de la ciudadanía y la voluntad política de las autoridades en todas las medidas para combatir la contaminación atmosférica en la ZMCM, se debe dar no solamente como una obligación, sino por convencimiento, teniendo en cuenta que la salud de la población es el beneficio primordial.

Los profesionales en el campo de la ingeniería ambiental no deben descuidar en ningún caso el aspecto técnico, así como principios fundamentales de prevención, minimización y control. Sin embargo, se debe entender que los aspectos económicos, sociales, culturales y políticos no están separados, y son dignos de tomarse en cuenta para lograr una calidad del aire satisfactoria a la cual tenemos derecho todos los mexicanos.

\section{Agradecimientos}

Los autores agradecen el apoyo brindado en la realización de los análisis de benceno a la Ing. Emma Bueno López, Dra. Laura González Ríos, Ing. Víctor Gutiérrez Avedoy del Centro Nacional de Investigación y Capacitación Ambiental (CENICA), y del Dr. Adrián Fernández del Instituto Nacional de Ecología. A los ingenieros José Luis Pedroza y Francisco Rivera, Rafael Ramos y Armando Retana del Gobierno del Distrito Federal (GDF) por la información de calidad del aire de la RAMA.

\section{Referencias}

Aldana T.P., Bravo A.H., Espinosa M.E., Sosa E.R., Tolivia M.E. y Torres B.R. (1999 a). Problemática de la contaminación atmosférica en la Zona Metropolitana de la Ciudad de México. Comité Técnico de Protección Ambiental. Revista del Instituto Mexicano de Ingenieros Químicos. Año XL, Vol. 5-6, 33-37. 
Aldana T.P., Bravo A.H., Espinosa M.E., Sosa E.R., Tolivia M.E., Torres B.R. (1999 b). Recomendaciones para mejorar la calidad del aire en la Zona Metropolitana de la Ciudad de México. Comité Técnico de Protección Ambiental. Revista del Instituto Mexicano de Ingenieros Químicos. Año XL, Vol. 7-9, 39-41.

SEDUE (1987-1988). Contaminación atmosférica por ozono: Propuesta de estrategias de control para el invierno 1987-1988 en la Zona Metropolitana de la Ciudad de México. Reporte Técnico elaborado para la Comisión Nacional de Ecología. SEDUE. México.

Bravo A.H., Sosa E.R. y Torres J.R. (1987). Contaminación atmosférica por ozono: Propuesta de estrategias de control para el invierno 1987-1988 en la Zona Metropolitana de la Ciudad de México. Reporte Técnico elaborado para la Comisión Nacional de Ecología. SEDUE. México.

Bravo A.H., Perrin F., Sosa E.R., Torres J.R. (1988). Incremento de la contaminación atmosférica por ozono en la zona metropolitana de la Ciudad de México. Ingeniería Ambiental, SMISAAC. Año 1. No. 1, 8-15.

Bravo A.H., Perrin F., Sosa E.R., Torres J.R. (1990). Efecto del cambio en la formulación en la gasolina sobre los niveles en la atmósfera de plomo y ozono en la Ciudad de México. En: Problemas de la cuenca de México. El Colegio Nacional.

Bravo A.H., Sosa E.R., Roy-Ocotla R., Sánchez A.P. (1993). Control Strategies vs. Meteorology Case. Mexico City. Paper A252. 86th Annual Meeting $\mathcal{E}$ Exhibition of the Air $\mathcal{E}$ Waste Management Association. Cincinnati, Ohio, June.

Bravo A.H., Sánchez A.P., Sosa E.R., Torres J.R. (1997 a). Air Pollution Problem in the Mexico City Metropolitan Zone: Photochemical Pollution. Paper FAl11.03. 90 th Annual Meeting E Exhibition of the Air $\mathcal{E}$ Waste Management Association. Toronto, Ontario, June.

Bravo A.H., Torres J.R., Sosa E.R. (1997 b). Reformulated Gasoline. The Experience of Mexico City. Paper $90^{\text {th }}$ Annual Meeting $E$ Exhibition of the Air $\mathcal{E}$ Waste Management Association. Toronto, Ontario, June.

Bravo A.H., Sosa E.R., Sánchez A.P., Jaimes P.M. (1998 a). New Ozone Standard in the USA
Applied to Mexico City. Paper $91^{\text {st }}$ Annual Meeting $\mathcal{E}$ Exhibition of the Air $\mathcal{E}$ Waste Management Association. San Diego, California, June.

Bravo A,H. Sosa E.R., Sánchez A.P., Jaimes P.M. (1998 b). The Dilemmas of the Change from Leaded to Unleaded Reformulated Gasolines in Developing Countries (Case: México). In $11^{\text {th }}$ World Clean Air and Environment Congress. South Africa. Vol. 4.

H. Bravo A., Sosa E.R., Sánchez A.P., Saavedra R.M. I., Jaimes P.M. (1999). Wildfires vs. Air Quality. Case Mexico City. Paper 99-623 to be presented $92^{\text {nd }}$ Annual Meeting $\mathcal{E}$ Exhibition of the Air $\varepsilon$ Waste Management Association. San Louis, Missouri, June.

Bravo A,H., Sosa E.R., Sánchez A.P. (2000 a). Atmospheric Pollution Problems Caused by Inadequate Use of Reformulated Gasolines (RFG) in Developing Countries. Third Especialty Conference on Environmental Progress in the Petroleum \& Petrochemical Industry. Saudi Arabian Section of the Air $\&$ Waste Management Association. Bahrein Society of Engineers. Bahrein, May.

Bravo A,H., Sosa E.R., Sánchez A.P., Jaimes P.M. (2000 b). Potential Urban Ozone Pollution as a Product of an Inadequate Implementation of a Best Available Control Technology (B.A.C.T.) in Developing Countries. 93 $3^{\text {rd }}$ Annual Conference $\varepsilon$ Exhibition of the Air \& Waste Management Association. Salt Lake City, Utah, June.

Bravo A,H., Sosa E.R., Sánchez A.P., Jaimes P.M., Lefhon A. (2001 a). The Piston Effect Observed in the Ozone Concentrations in the Mexico City Metropolitan Zone. 94th Annual Conference $\mathcal{E}$ Exhibition of the Air $\mathcal{E}$ Waste Management Association. Orlando, Florida, June.

Bravo A.H., Sosa E.R., Sánchez A.P., Bueno L.E., González R.L. (200l b). Concentrations of Benzene and Toluene in the Atmosphere of the Southwestern Area at the Mexico City Metropolitan Zone. A presentarse en: $12^{\text {th }}$ World Clean Air E Environment Congress and Exhibition. 26-31 August, Seoul, Korea.

Bravo A.H., Sosa E.R., Sánchez A.P., Jaimes P.M., Saavedra R.M.I. (2001 C) Wildfires Impact on the Air Quality of Mexico City, 1992-1999. Aceptado 
en: Environmental Pollution. Elsevier. ISSN 0269-7491.

Chow P.S. (1990). Consecuencias inmediatas del uso indiscriminado de gasolinas sin plomo en la Zona Metropolitana de la Ciudad de México. En: Problemas de la cuenca de México. El Colegio Nacional.

DDF (1989). Programa integral contra la contaminación atmosférica en el Valle de México. Departamento del Distrito Federal.

DDF, GEM, SEMARNAP, SSA (1996) Programa para Mejorar la Calidad del Aire en el Valle de México 1995-2000 (PROAIRE). Departamento del Distrito Federal, Gobierno del Estado de México, Secretaría de Medio Ambiente, Recursos Naturales y Pesca, Secretaría de Salud.

EA (1999). Administration of Air Pollution Control. Environmental Agency. Air Quality Bureau. Japan. EPAOS (1991). Benzene, Expert Panel on Air Quality Standards, Department of the Environment. United Kingdom.
INE, CENICA (1996). Primer informe sobre la calidad del aire en ciudades mexicanas. Instituto Nacional de Ecología, Centro Nacional de Investigación y Capacitación Ambiental.

Mehlman M.A. (1994). Dangerous and Cancer-Causing Properties of Products and Chemicals in the Oil Refining and Petrochemical Industry-Part XX: Health Dangers of Petroleum Hydrocarbons: Gasoline, Methyl-Tertiary Butyl Ether, Benzene, 1,3, Butadiene and Alkylbenzenes. Journal of Clean Technology and Environmental Sciences. 4: 37-57.

Sosa E.R. (2001). Evaluación y control de benceno en la Zona Metropolitana de la Ciudad de México. Tesis de Doctorado en Ingeniería. División de Estudios de Posgrado, Facultad de Ingeniería, UNAM

Vega, R.E., Mora, P.V., Mugica, A.V. (1998). Particulate Matter Pollution in Mexico City, Proceedings of the 9 I $^{\text {st }}$ Annual Meeting $\mathcal{E}$ Exhibition of the air E Waste Management Associations, San Diego, California.

\section{Semblanza de los autores}

Humberto Bravo-Alvarez. Realizó la licenciatura en química y la maestría en química nuclear en la UNAM.. Posteriormente obtuvo el grado de doctor en ingeniería en la Universidad de West Virginia. Es investigador del Centro de Ciencias de la Atmósfera de la UNAM, en donde se desempeña como jefe de la Sección de Contaminación Ambiental desde 1977. Su área de interés por más de 30 años ha sido la contaminación y la ingeniería ambiental. Es profesor de la maestría en ingeniería ambiental en la División de Estudios de Posgrado de Facultad de Ingeniería (DEPFI) de la UNAM desde 1988. Es perito colegiado del Colegio Nacional de Ingenieros Químicos y de Químicos (CONIOO) en protección ambiental, así como Qualified Environmental Profesional (QEP) del Institute of Professional Environmental Practice.

Rodolfo Sosa-Echeverria. Realizó su licenciatura en ingeniería química, maestría en ingeniería ambiental, y doctorado en ingeniería en la UNAM. Es técnico académico de la Sección de Contaminación Ambiental en el CCA desde 1986. Ha sido profesor de la DEPFI desde 1988. Es perito colegiado del CONIQQ en protección ambiental y fue presidente del Capítulo México de la Air \& Waste Management Association de 1991 a 1993.

Pablo Sánchez-Alvarez. Realizó la licenciatura en biología en la Facultad de Ciencias de la UNAM. Actualmente es técnico académico en la Sección de Contaminación Ambiental del CCA UNAM, además de ser responsable de la operación y mantenimiento de la estación de monitoreo automático de contaminantes criterio y depositación ácida, es instructor certificado en la línea de investigación de la atmósfera y el clima del Programa Internacional de Aprendizajes y Observaciones Globales en Beneficio del Medio Ambiente (The GLOBE Program México).

Mónica Jaimes-Palomera. Estudió la licenciatura en biología en la Escuela Nacional de Estudios Profesionales - Iztacala, UNAM, posteriormente realizó la especialidad en estadística aplicada en el IIMAS de la UNAM, finalmente realizó sus estudios de maestría en ingeniería ambiental en la División de Estudios de Posgrado de la Facultad de Ingeniería de la UNAM. Participó en proyectos de ecología de la conducta con el Dr. Hugh Drummond en el Instituto de Ecología de la UNAM de 1985 a 1990. Ha participado en proyectos de la Sección de Contaminación Ambiental del CCA a cargo del Dr. Humberto Bravo. Actualmente trabaja en la Secretaría del Medio Ambiente del GDF, en el área de Análisis e Información, desarrollando proyectos estadísticos de calidad. 